\section{Microwave Electronics}

\section{Hartwig Thim zur Emeritierung}

Herr O. Univ.-Prof. Dipl.-Ing. Dr. techn. Hartwig Thim vollendete am 18. März 2003 sein 68. Lebensjahr und emeritiert mit Ende September dieses Jahres. Er beendet damit seine fast achtzehnjährige Tätigkeit in Linz als Vorstand des Instituts für Mikroelektronik der Johannes Kepler Universität und übergibt ein umfangreiches Lebenswerk, das der Mikroelektronik und der Höchstfrequenztechnik gewidmet war und ist.

Die in diesem e\&i-Heft vorliegenden Beiträge zum Thema "Microwave Electronics" sind Prof. Thim gewidmet. Ein weiteres Schwerpunktheft zu Ehren Prof. Thim erscheint als Jänner-Ausgabe 2004 zum Thema "Microwave Fields and Applications".

Hartwig Thim wurde in Wels geboren, wuchs in Linz auf und studierte an der Technischen Universität Wien Elektrotechnik (Diplom 1960 und Doktorat 1964). Nach der Sponsion zum Dipl.-Ing. im Jahre 1960 war er bis zum Abschluss seiner Doktorarbeit zum Thema "Dielektrische Sphäroidantenne“ Universitätsassistent am Institut für Grundlagen und Theorie der Elektrotechnik an der TU Wien.

Seine weitere berufliche Laufbahn führte inn dann nach Abschluss seines Doktorates in die Vereinigten Staaten von Amerika an die Solid State Electronics-Abteilung der AT\&T Bell Telephone Laboratories in Murray Hill, New Jersey, USA. Internationale Beachtung fand Professor Thim durch die Entwicklung des ersten stabilen Halbleiter-Verstärkers im Mikrowellenbereich (Transferred Electron Amplifier, TEA). Dieser TEA basierte auf dem Gunn-Effekt, einer damals noch jungen Entdeckung, der sich Professor Thim in den vielen Jahren seiner wissenschaftlichen Karriere immer wieder intensiv widmete. Dieses Engagement führte unter anderem dazu, dass die stabile Betriebsart dieses Halbleiterbauelements nach inm benannt wurde. Im Jahre 1969 wurde Professor Thim zum Leiter der Abteilung Mikrowellen am Fraunhofer-Institut für Angewandte Festkörperphysik in Freiburg, Deutschland bestellt. Fünf Jahre später wurde er zum Ordentlichen Professor für Industrielle Elektronik an der TU Wien berufen, und im Jahre 1985 schließlich folgte er dem Ruf an die Johannes Kepler Universität Linz.

Neben seiner wissenschaftlichen Arbeit engagierte sich Professor Thim auch in der akademischen Selbstvenwaltung und der Weiterentwicklung der Universität Linz. Besonders hervorzuheben sind hier seine fruchtbaren Beiträge zur Gründung und Gestaltung des neuen Studiengangs Mechatronik Ende der

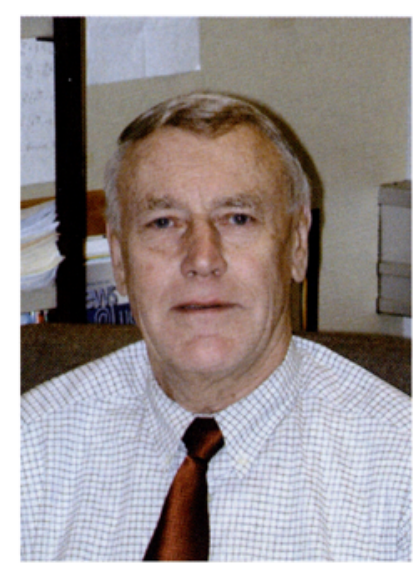

Prof. Hartwig Thim vollendete im März sein 68. Lebensjahr

achtziger Jahre und seine Aktivitäten als Dekan der TechnischNaturwissenschaftlichen Fakultät in den Jahren 1992 bis 1994. Sein Einsatz und seine gewissenhafte Amtsführung brachten inm den Respekt und Dank der Fakultät ein.

In seinen aktuellen Forschungsarbeiten beschäftigt sich Professor Thim heute insbesondere mit speziellen Halbleiterbauelementen zur Erzeugung von Millimeterwellen wie den HEIFET. Des Weiteren arbeitet er intensiv daran, seine Zweifel an der Richtigkeit der Einsteinschen Speziellen Relativitätstheorie nachzuweisen. Abseits der Wissenschaft widmet er sich neben seiner Familie und dem Freundeskreis mit großer Leidenschaft dem Fußballspiel, das er trotz einer vor einigen Jahren erfolgten Knieoperation nach wie vor allwöchentlich betreibt.

Emeritierung bedeutet, von den Aufgaben in Lehre und Verwaltung entlastet zu sein. Sie bedeutet daher auch, sich der Forschung und den schönen Seiten des Lebens stärker widmen und seinen Hobbys Priorität einräumen zu können.

In diesem Sinne und im Namen seiner derzeitigen und ehemaligen Kollegen und Mitarbeiter und persönlich wünschen wir inm Gesundheit und ein weiterhin erfülltes Leben.

\section{Andreas Springer}

Andreas Stelzer OVE

Robert Weigel OVE 